\title{
Dynamic Programming for Minimal Cost Topology with Reliability Constraint
}

\author{
Basima Elshqeirat, Sieteng Soh, Suresh Rai, and Mihai Lazarescu
}

\begin{abstract}
This paper addresses an NP-hard problem, called NTD-CR, to design a minimal-cost communication network topology that satisfies a pre-defined reliability constraint. Since reliability is always a major issue in the network design, the problem is practical for critical applications requiring minimized cost. The paper formulates a dynamic programming (DP) scheme to solve NTD-CR problem. DP approach, called DPCR-ST, generates the topology using a selected set of spanning trees of the network, STX $_{\min }$. We propose three greedy heuristics to generate and order only $k$ spanning trees of the network. Each heuristic allows DPCR-ST to enumerate STX min $_{\text {in }}$ using only $k$ spanning trees, which improves the time complexity while producing near optimal topology. Simulations based on fully connected networks that contain up to $2.3 \times 10^{9}$ spanning trees show the merits of ordering methods and the effectiveness of our algorithm vis-à-vis four existing state-of-the-art techniques; DPCR-ST produces $81.5 \%$ optimal results, while using only $0.77 \%$ of the spanning trees contained in network.
\end{abstract}

Index Terms-Dynamic programing, network optimization, network reliability, network topology design.

\section{INTRODUCTION}

A well-designed communication network is inseparable from the effective running of user applications. For critical applications (e.g., emergency system, rescue and military operations) it is important that the communication network topology is as reliable as possible since in practice network components (e.g., links) are failure-prone. A more reliable topology will make the communication network operate effectively and without interruption, even in the presence of the component failures [1].Further, some applications may need to run on a topology with a guaranteed minimum reliability, $R_{\min }$, to properly operate. However, constructing a reliable topology incurs higher installation cost. Given a set of various centers (nodes), their possible connecting links, link failure rate and installation cost, NTD-CR selects the most suitable set of links such that the resulting model meets its required reliability $R_{\min }$ while minimizing its installation cost. This paperconsiders network reliability [2], also called all-terminal reliability, as the measure of reliability.

The NTD-CR problem has been shown NP-hard [3], and thus one must use heuristic and/or approximation solutions to design large sized topologies. There are many proposed

Manuscript received February 20, 2013; revised August 1, 2013

B. Elshqeirat, S. Soh, and M. Lazarescu are with Department of Computing, Curtin University, Perth, Western Australia; (e-mail: Basima.elshoqeirat@postgrad.curtin.edu.au, S.Soh@curtin.edu.au, M.Lazarescu@curtin.edu.au; tel.: +61 89266 2984; fax: +61 89266 2819).

S. Rai is with Department of Electrical and Computer Engineering, Louisiana State University, Baton Rouge, LA, USA. (e-mail: srai@1su.edu). solutions for NTD-CR problem. The existing algorithms that generate approximation solution are mainly based on meta-heuristic techniques, e.g., Genetic Algorithm [2], [3], Swarm Particle [4] and Ant Colony [5]. While the metaheuristic algorithms can significantly reduce time complexity, they still require numerous iterations to converge and thus use a considerable computational effort while producing only up to $63.1 \%$ optimal solutions. Thus, approach that can produce better results is still needed, especially for use in large scale networks.

The main contribution of this paper is two folds. First, it uses a dynamic programming (DP) formulation to generate topology based on the proposed algorithm, DPCR-ST. Second, this paper proposes three heuristics to enumerate only $k \leq n$ spanning trees, which are used by DPCR-ST to significantly reduce its time complexity; $n$ is the total number of spanning trees in the network.

The layout of this paper is as follows. Section II discusses the network model and notations. Section III formulates the NTD-CR problem and provides assumptions. Section IV describes our proposed solutions while Section V presents the simulation results. Finally, Section VI concludes the paper and discusses the future work.

\section{NETWORK MODEL AND NOTATIONS}

A communication network can be modeled by a probabilistic bidirectional simple graph $\mathrm{G}=(\mathrm{V}, \mathrm{E})$, in which each vertex/node $\mathrm{v}_{i} \in \mathrm{V}$ represents a network component (e.g., router or computer site) and each edge $\mathrm{e}_{j} \in \mathrm{E}$ represents the connecting media (e.g., cable or communication link) between the network components. It is assumed that all node locations and connecting links are given. Each $\mathrm{e}_{j}$ has a cost $\mathrm{c}_{j}>0$ that represents the cost to install $\mathrm{e}_{j}$, and reliability $0 \leq \mathrm{r}_{j} \leq 1$ that represents the probability that $\mathrm{e}_{j}$ is functioning (UP); all nodes are always UP and use no setup costs. Edge failures are assumed statistically independent and without repair. Fig. 1 (a) shows an example of the graph model of a network with four fixedly positioned nodes and five links; Table I provides $\operatorname{cost}\left(\mathrm{c}_{j}\right)$ and reliability $\left(\mathrm{r}_{j}\right)$ values for an edge $\mathrm{e}_{j}$.

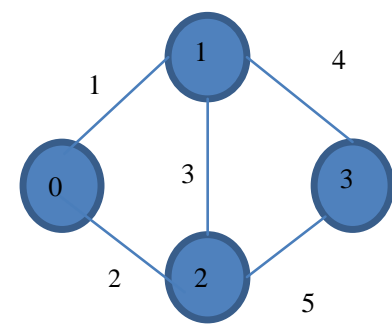

(a)

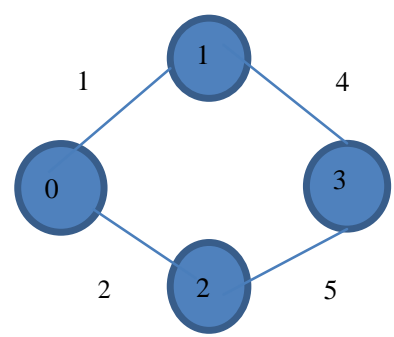

(b)
Fig. 1. An example network and optimal solution.

A spanning tree $i, \mathrm{ST}_{i}$, is a subgraph of $\mathrm{G}$, which is a tree and contains all vertices in G. A spanning tree in a network 
with $|\mathrm{V}|$ nodes has $(|\mathrm{V}|-1)$ links. Let $\mathrm{ST}_{G}$ be a set of all spanning trees in $\mathrm{G}, n=\left|\mathrm{ST}_{G}\right|$, and $\mathrm{L}_{i}$ be the set of links in $\mathrm{ST}_{i} \in \mathrm{ST}_{G}$. Table I shows $\mathrm{ST}_{G}$ of the network in Fig. 1 (a).

TABLE I: LiNK WEIGHT AND SPANNING TREE SET FOR NETWORK IN FIG. 1 (A)

\begin{tabular}{|c|c|c|c|c|c|c|}
\hline & \multicolumn{3}{|c|}{$\mathrm{ST}_{G}$} & \multicolumn{3}{|c|}{ Link Weight } \\
\hline$i$ & $\mathrm{ST}_{i}$ & $\operatorname{Rel}\left(\mathrm{ST}_{i}\right)$ & $\operatorname{Cost}\left(\mathrm{ST}_{i}\right)$ & $\mathrm{e}_{j}$ & $\mathrm{c}_{j}$ & $\mathrm{r}_{j}$ \\
\hline 1 & $(1,3,5)$ & 0.567 & 13 & 1 & 5 & 0.9 \\
\hline 2 & $(1,3,4)$ & 0.567 & 11 & 2 & 3 & 0.6 \\
\hline 3 & $(2,3,4)$ & 0.378 & 9 & 3 & 2 & 0.7 \\
\hline 4 & $(1,2,5)$ & 0.486 & 14 & 4 & 4 & 0.9 \\
\hline 5 & $(2,3,5)$ & 0.378 & 11 & 5 & 6 & 0.9 \\
\hline 6 & $(2,1,4)$ & 0.486 & 12 & & & \\
\hline 7 & $(1,4,5)$ & 0.729 & 15 & & & \\
\hline 8 & $(2,5,4)$ & 0.486 & 13 & & & \\
\hline
\end{tabular}

Let $\operatorname{Cost}\left(\mathrm{ST}_{i}\right)$ denote the cost of installing all links in spanning tree $\mathrm{ST}_{i}$, computed by taking the sum of $\mathrm{c}_{j}$ of each $\mathrm{e}_{j}$ in $\mathrm{ST}_{i}$. The cost of a network topology $\mathrm{G}, \operatorname{Cost}(\mathrm{G})$, is obtained using the sum of all $c_{j}$ for each $\mathrm{e}_{j} \mathrm{in} \mathrm{G}$. Let $\operatorname{Rel}\left(\mathrm{ST}_{i}\right)$ denote the reliability of spanning tree $\mathrm{ST}_{i}$; it is calculated by multiplying all $\mathrm{r}_{j}$ of each $\mathrm{e}_{j}$ in $\mathrm{ST}_{i}$. The network reliability of a topology $\mathrm{G}, \operatorname{Rel}(\mathrm{G})$, is the probability that at least one $\mathrm{ST}_{i}$ in $\mathrm{G}$ is functional. In another word, it is the probability that a set of operational links provides communication path between every pair of nodes. Calculating $\operatorname{Rel}(\mathrm{G})$, in general, is an NP-hard problem [5]; Section III.B provides details about computing $\operatorname{Rel}(\mathrm{G})$. Notice that $\mathrm{G}$ can be constructed using nodes in $\mathrm{V}$ and all links in $\mathrm{ST}_{G}$, and thus this paper

$$
\text { uses } \operatorname{Cost}\left(\mathrm{ST}_{G}\right)=\operatorname{Cost}(\mathrm{G})=\operatorname{Cost}(\mathrm{E})
$$

and

$$
\operatorname{Rel}\left(\mathrm{ST}_{G}\right)=\operatorname{Rel}(\mathrm{G})=\operatorname{Rel}(\mathrm{E})
$$

Similarly, we consider that

$$
\operatorname{Cost}\left(\mathrm{ST}_{i}\right)=\operatorname{Cost}\left(\mathrm{L}_{i}\right)
$$

and

$$
\operatorname{Rel}\left(\mathrm{ST}_{i}\right)=\operatorname{Rel}\left(\mathrm{L}_{i}\right)
$$

\section{NETWORK DESIGN PROBLEM AND SOLUTION}

Let $X_{i}$ be a decision variable $\{0,1\}$ that indicates if spanning tree $\mathrm{ST}_{i}$ in Gis selected $\left(X_{i}=1\right)$ or not selected $\left(X_{i}=0\right)$. The following equations describe the NTD-CR problem.

$$
\begin{aligned}
& \text { Minimize } \operatorname{Cost}\left(\bigcup_{\substack{i=1 \\
\left|S T_{G}\right|}}^{\left|S T_{G}\right|} S T_{i} X_{i}\right) \\
& \text { Subject to } \operatorname{Rel}\left(\bigcup_{i=1}^{\mid} S T_{i} X_{i}\right) \geq R_{\text {min }}
\end{aligned}
$$

Equation (1) calculates the minimum cost of the network using only the selected spanning trees $\mathrm{ST}_{i}$ from (2). One may generate all $2^{n}$ possible combinations of spanning trees that meet the constraint in(2). Then, for each combination that has reliability at least $R_{\min }$, use(1) to calculate its cost and select the topology with the minimum cost as $G_{\min }$ with $\operatorname{Rel}\left(G_{\text {min }}\right) \geq R_{\text {min }}$. This solution is prohibitive for use in large networks since a general network contains $n=O\left(|\mathrm{~V}|^{|\mathrm{V}|}\right)$ spanning trees [6]. In Section IV.A, we propose aDP approach to solve (1) and (2).
To illustrate the NTD-CR problem, consider the network in Fig. 1 (a). For $R_{\min }=0.87$, Fig. 1 (b) shows the optimal network topology, $G_{\min }$, whose links form a set of spanning $\operatorname{trees}\{(2,5,4),(1,4,5),(2,1,4),(1,2,5)\}$ with $\operatorname{Rel}\left(G_{\text {min }}\right)=0.88$ and $\operatorname{Cost}\left(G_{\text {min }}\right)=18 ; G_{\text {min }}$ does not contain spanning trees $(1,3,4),(1,3,5),(2,3,5)$ and $(2,3,4)$ because link 3 is not selected.

\section{Proposed DYNAMIC PROGRAMMING-BASED SOLUTION}

\section{A. Dynamic Programming Formulation for NTD-CR}

Let $\mathrm{STX}_{i}$, for $i=1,2, \ldots, n$, be a set of spanning trees selected from $n-i+1$ spanning trees in $\left\{\mathrm{ST}_{i}, \mathrm{ST}_{i+1}, \ldots, \mathrm{ST}_{n}\right\}$ and $\mathrm{G}_{i}=\left(\mathrm{V}, \mathrm{E}_{i} \subseteq \mathrm{E}\right)$ be its induced graph whose links comprise of all links in $\mathrm{STX}_{i}$. We use $\mathrm{STX}_{i}$ and $\mathrm{G}_{i}$ interchangbly since one can be generated from the other. Note that $0 \leq\left|\mathrm{STX}_{i}\right| \leq n-i+1$, and there are $2^{n}$ different $\mathrm{STX}_{i}$, and we aim to select $\mathrm{STX}_{1}$ with a reliability of at least $R_{\text {min }}$, i.e., $\operatorname{Rel}\left(\mathrm{G}_{1}\right) \geq R_{\text {min }}$ and minimum $\operatorname{Cost}\left(\mathrm{G}_{1}\right)$.

Let DP[1.. $n, 0 . . \check{\mathrm{R}}_{\text {min }}$ ] be a 2-dimension DPtable, where $\check{\mathrm{R}}_{\text {min }}=\operatorname{round}\left(\delta \times R_{\text {min }}\right)$, for a positive integer multiplier $\delta$ and a function $\operatorname{round}(\bullet)$ that returns the closest integer value of $(\bullet)$. For example, the function returns $\breve{\mathrm{R}}_{\text {min }}=92\left(\breve{\mathrm{R}}_{\min }=93\right)$ when we set $\delta=100$ and $R_{\text {min }}=0.9216\left(R_{\min }=0.9261\right)$.

Each element DP $[i, \check{r}]$, for $i=1,2, \ldots, n, \check{r}=0,1,2, \ldots$, $\check{\mathrm{R}}_{\text {min }}$, stores five pieces of information: a cost $\mathrm{C}[i, \check{r}]>0$, a reliability $0 \leq \mathrm{R}[i, \check{r}] \leq 1.0, \mathrm{ST}[i, \check{r}] \subseteq \mathrm{ST}_{\mathrm{G}}$, a set of links $\mathrm{L}[i$, $\check{r}] \subseteq \mathrm{E}$, and an integer index $0 \leq J[i, \check{r}] \leq \delta$. In essence, the columns of DP table partition the reliability constraint $R_{\min }$ into $\delta$ consecutive reliability constraints, i.e., $R_{\min } / \delta$, $\left(2 \times R_{\min }\right) / \delta, \ldots, \delta \times R_{\min } / \delta=R_{\min }$. In other words, each column index $\check{r}=0,1, \ldots, \breve{\mathrm{R}}_{\min }$, corresponds to a reliability constraint $r=0,1 / \delta, \ldots,\left(\check{\mathrm{R}}_{\text {min }} / \delta\right) \approx R_{\text {min }}$, i.e., $r=\check{r} / \delta$ and $\check{r}=\operatorname{round}(\delta \times r)$, and each DP $[i, \dot{r}]$ is used to store four pieces of information of each selected topology $\mathrm{G}_{i}$ that has $\operatorname{Rel}\left(\mathrm{G}_{i}\right) \geq r$. Specifically, for each $\operatorname{Rel}\left(\mathrm{G}_{i}\right) \geq r$, we set $\mathrm{C}[i, \grave{r}]=\operatorname{Cost}\left(\mathrm{G}_{i}\right), \mathrm{R}[i, \grave{r}]=\operatorname{Rel}\left(\mathrm{G}_{i}\right), \mathrm{ST}[i$, $\check{r}]=\mathrm{STX}_{\mathrm{i}}$, and $\mathrm{L}[i, \check{r}]=\mathrm{E}_{i}$. For $\operatorname{Rel}\left(\mathrm{G}_{i}\right)<r$, we set $\mathrm{C}[i, \check{r}]=\infty, \mathrm{R}[i$, $\check{r}]=0, \mathrm{ST}[i, \check{r}]=\{\}$, and $\mathrm{L}[i, \check{r}]=()$. Note that $\mathrm{C}[i, \check{r}]=0$ is not possible since each link is assumed to have a non-zero cost. Since $C\left[1, \check{\mathrm{R}}_{\text {min }}\right]$ is the cost of $\mathrm{G}_{1}=\left(\mathrm{V}, \mathrm{E}_{1} \subseteq \mathrm{E}\right)$ with $\operatorname{Rel}\left(\mathrm{G}_{1}\right) \geq R_{\text {min }}, \mathrm{NTD}-\mathrm{CR}$ aims to generate $\mathrm{DP}\left[1, \breve{\mathrm{R}}_{\text {min }}\right]$ that contains the minimum $\mathrm{C}\left[1, \breve{\mathrm{R}}_{\text {min }}\right]$, which represent the $\mathrm{G}_{\text {min }}$.

For each range of columns $\check{r} 1 \leq \check{r} \leq \check{r} 2$ in row $i$ that contain the same reliability value, we set each $J[i, \check{r}]=\check{r} 2$. Thus, index $J[i, r]=0,1,2, \ldots, 100$ marks the ending column of a range of columns that have the same reliability. For example, we store $J[i, \check{r}]=38$ at columns $\check{r}=0$ to $\check{r}=38$ if $\mathrm{R}[i, 0]=\mathrm{R}[i, 1]=\ldots=\mathrm{R}[i$, 38]. Note that we set $J[i, \check{r}]=\check{r}$ when $\check{r} 1=\check{r} 2$, i.e., when the length of the range is one.

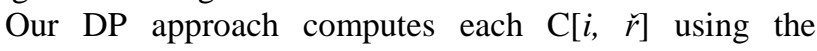
following four equations:

$i=n$ :

$$
\begin{gathered}
\mathrm{C}[i, \check{r}]=\operatorname{Cost}\left(\mathrm{ST}_{i}\right) ; \text { if } \operatorname{Rel}\left(\mathrm{ST}_{i}\right) \geq r \\
\mathrm{C}[i, \check{r}]=\infty ; \text { if } \operatorname{Rel}\left(\mathrm{ST}_{i}\right)<r
\end{gathered}
$$

$i<n$ and $\operatorname{Rel}\left(\mathrm{ST}_{i}\right) \geq r$ :

$$
\mathrm{C}[i, \check{r}]=\operatorname{Min}\left(\mathrm{C}[i+1, \check{r}], \operatorname{Cost}\left(\mathrm{ST}_{i}\right)\right)
$$


$i<n$ and $\operatorname{Rel}\left(\mathrm{L}[i+1, j] \cup \mathrm{L}_{i}\right) \geq r:$

$$
\mathrm{C}[i, \check{r}]=\operatorname{Min}\left(\mathrm{C}[i+1, \check{r}], \operatorname{Cost}\left(\mathrm{L}[i+1, j] \cup \mathrm{L}_{i}\right)\right)
$$

Without loss of generality, we consider the spanning tree selection start from the last spanning tree $\mathrm{ST}_{n}$. In (3), when the last spanning tree has reliability of at least $r$, it should be selected, giving $\mathrm{C}[n, \check{r}]=\operatorname{Cost}\left(\mathrm{ST}_{n}\right)$. In contrast, when $\operatorname{Rel}\left(\mathrm{ST}_{n}\right)<r, \mathrm{ST}_{n}$ is not selected because it does not meet the constraint $r$; thus (4) sets $\mathrm{C}[n, \check{r}]=\infty$ to denote that no spanning tree is selected.

Equation (5) and (6) are used for each remaining $\mathrm{ST}_{i}$, for $i=n-1, n-2, \ldots, 1$. Equation (5) considers two options, selecting or not selecting $\mathrm{ST}_{i}$, when $\operatorname{Rel}\left(\mathrm{ST}_{i}\right) \geq r$, and selects the option that produces the minimum cost. Specifically, when $\mathrm{ST}_{i}$ is selected (not selected), its cost is $\operatorname{Cost}\left(\mathrm{ST}_{i}\right)$ $(\mathrm{C}[i+1, \check{r}])$, and the equation selects the minimum between the two since both options satisfy the reliability requirement $r$. Note that the reliability value in the element would be changed to $\operatorname{Rel}\left(\mathrm{ST}_{i}\right)$ if $\mathrm{ST}_{i}$ is selected. Further, (5) considers a situation when no trees have been selected for column $\check{r}$, i.e., $\mathrm{C}[i+1, \check{r}]=\infty$ and $\mathrm{R}[i+1, \check{r}]=0$, in which case it will select $\mathrm{ST}_{i}$.

Equation (6) considers the case when selecting $\mathrm{ST}_{i}$ together with some previously selected trees $\mathrm{STX}_{j}$ satisfies the required reliability $r$, i.e., $\operatorname{Rel}\left(\mathrm{L}[i+1, j] \cup \mathrm{L}_{i}\right) \geq r$, for each possible $j=J[i, \check{r}]=0,1, \ldots, 100$. Like (5), (6) also considers the minimum cost between either selecting or not selecting $\mathrm{ST}_{i}$; the former produces $\operatorname{Cost}\left(\mathrm{L}[i+1, j] \cup \mathrm{L}_{i}\right)$ and the latter $\mathrm{C}[i+1$, r]. Specifically, when $\mathrm{ST}_{i}$ is selected (not selected), the cost is calculated from the selected spanning trees $\mathrm{STX}_{i},\left(\mathrm{STX}_{i+1}\right)$. Note that the reliability value in the column would be changed to $\operatorname{Rel}\left(\mathrm{L}[i+1, j] \cup \mathrm{L}_{i}\right)$ if $\mathrm{ST}_{i}$ is selected. Further, (6) also considers a situation when no trees have been selected for column $\check{r}$, i.e., $\mathrm{C}[i+1, \check{r}]=\infty$ and $\mathrm{R}[i+1, \check{r}]=0$, in which it will select $\mathrm{ST}_{i}$.

The DP formulation in (3) to (6) is similar to the DP solution for the well-known NP-complete 0/1 knapsack problem [7]. In the $0 / 1$ knapsack problem, there are $n$ items where each item has capacity and value and its goal is to select a set of items that have the maximumtotal value while having total capacityno larger than a given capacity constraint. In contrast, NTD-CR aims to select a set of spanning trees whose induced topology has minimumtotal cost while having network reliability no less than a given reliability constraint $R_{\text {min }}$. However, unlike for knapsack where the total cost of two items is the sum of each item's cost, in NTD-CR, $\operatorname{Cost}\left(\mathrm{ST}_{i}\right)+\operatorname{Cost}\left(\mathrm{ST}_{p}\right) \geq \operatorname{Cost}\left(\mathrm{ST}_{i} \cup \mathrm{ST}_{p}\right)$ because $\mathrm{ST}_{i}$ and $\mathrm{ST}_{p}$ may contain common links. Therefore (6) must consider all possible values of $j$, i.e., $J[i, r]$. Further, while the total capacity of two items in Knapsack equals the sum of each item's capacity, in NTD-CR, $\operatorname{Rel}\left(\mathrm{ST}_{i}\right)+\operatorname{Rel}\left(\mathrm{ST}_{p}\right) \neq \operatorname{Rel}\left(\left\{\mathrm{ST}_{i} \cup \mathrm{ST}_{p}\right\}\right)$, and $\operatorname{Rel}\left(\mathrm{ST}_{i}\right)>\operatorname{Rel}\left(\mathrm{ST}_{p}\right)$ does not always mean $\operatorname{Rel}\left(\mathrm{ST}_{h} \cup \mathrm{ST}_{i}\right)>\operatorname{Rel}\left(\mathrm{ST}_{h} \cup \mathrm{ST}_{p}\right)$, for any $\mathrm{ST}_{h}$. Therefore, each $\mathrm{C}[i, \dot{r}]$ is not necessarily minimum even when it is computed from two optimal sub problems.

\section{B. DPCR-ST Algorithm}

Fig. 2 shows our proposed DP algorithm, called DPCR-ST, that directly applies (3) to(6).For a $\mathrm{G}=(\mathrm{V}, \mathrm{E})$ that contains $n$ spanning trees with reliability constraint $R_{\text {min }}$, DPCR-ST implicitly constructs a DP table of size $n \times \check{\mathrm{R}}_{\min }$. As shown in
Fig. 2, DPCR-ST keeps only two consecutive rows, called row 1 and row2, and therefore it requires only a table of size $2 \times \check{\mathrm{R}}_{\text {min }}$. Specifically, DPCR-ST computes C $[1, j]$ and R $[1, j]$ in row 1 using the information in $\mathrm{C}[2, \dot{r}]$ and $\mathrm{R}[2, \dot{r}]$ in row 2 , for all relevant columns $\check{r}$ and $j$. After copying the contents of row 1 to row 2 , it repeats the step until all spanning trees are considered.

Line 1 implements (4) while Line 2 to 8 are based on (3). The remainder of the code is used to implement (5) and (6). Specifically, (5) is solved in Line 9 to $21,(6)$ in Line 22 to 38 , and Line 39 to 45 copies the contents of row 1 to row2.

\section{DPCR-ST Analysis}

The time complexity of DPCR-ST can be computed as follows.The $\operatorname{Cost}(\mathrm{X})$ function requires all unique links in the set of spanning trees $X$.

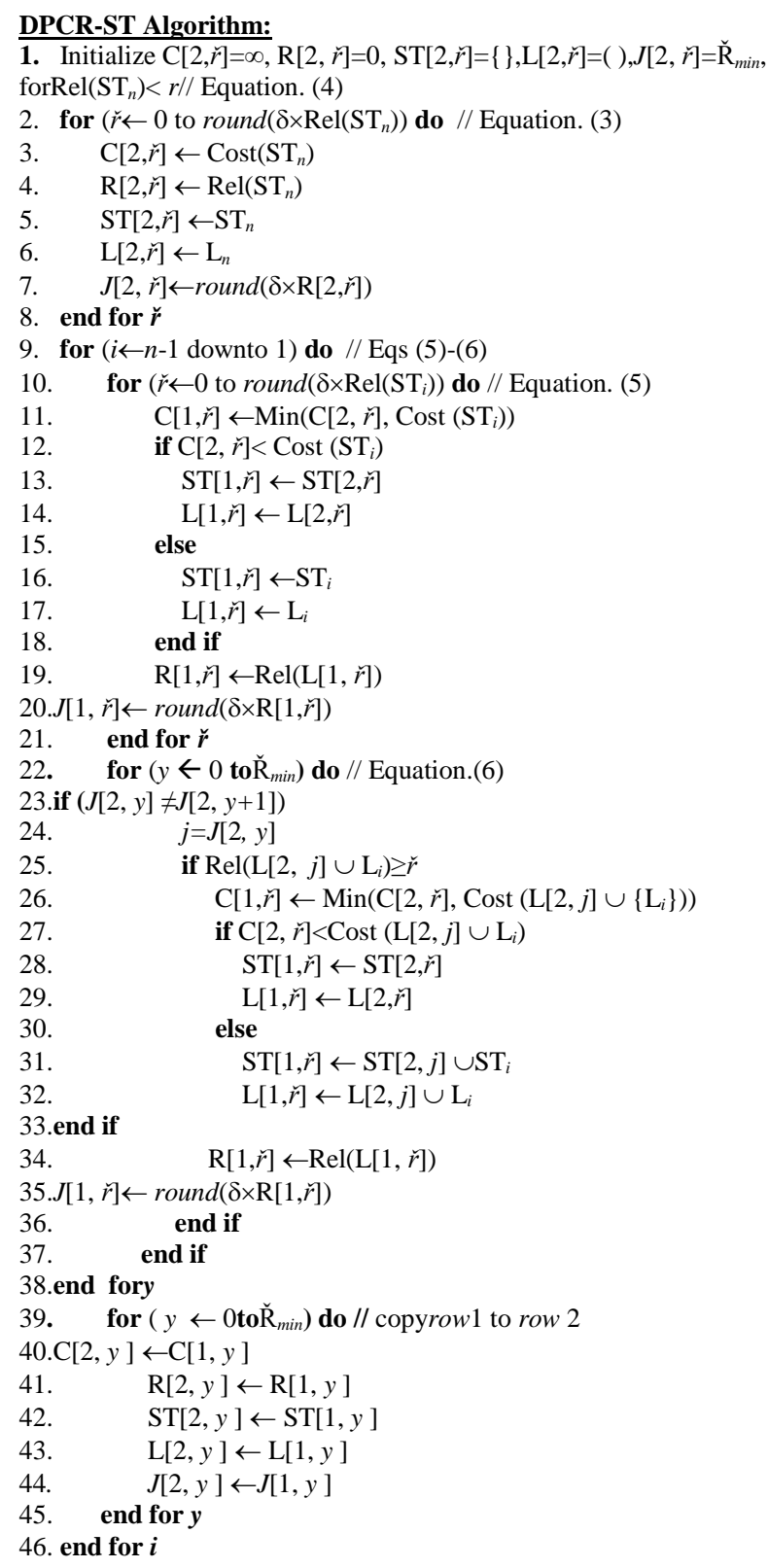

Fig. 2. DPCR-ST Pseudocode

For each $\check{r}, \operatorname{Cost}(\mathrm{X})$ returns the sum of $\mathrm{C}[i+1, \check{r}]$ and the cost of links in $\mathrm{ST}_{i}$ that are not inL $[i+1, \check{r}]$. Using the bit 
implementation [8], one requires only one bit OR and one bit XOR operation to obtain the links in $\mathrm{ST}_{i}$ that are not $\operatorname{inL}[i+1, \check{r}]$, and thus for any $\mathrm{X}, \operatorname{Cost}(\mathrm{X})$ can be computed in $O(|\mathrm{E}|)$. DPCR-ST uses the function at most once for every table entry, and therefore the worst case time complexity for using the function is $O\left(n \times|\mathrm{E}| \times \check{\mathrm{R}}_{\text {min }}\right)$.

The $\operatorname{Rel}(\mathrm{X})$ function can be implemented using any exact reliability calculation [8], heuristic technique [9] or approximation (bounding) method [2]. In this paper, we use Monte Carlo simulation [9] with time complexity $O\left(b \times|\mathrm{V}|^{4}\right)$ [1] to estimate $\operatorname{Rel}(\mathrm{X})$ of each candidate network; $b$ is the number of replication. Notice that $\operatorname{Rel}(\mathrm{X})$ is used only for each different $j$ in each row $i$. Hence, in total, the time complexity of using $\operatorname{Rel}(\mathrm{X})$ is $O\left(\psi \times b \times|\mathrm{V}|^{4}\right)$, where $\psi$ is the total number of different $j$ in the table. Thus, in the worst case, DPCR-ST requires $O\left(\psi \times b \times|\mathrm{V}|^{4}+n \times|\mathrm{E}| \times \check{\mathrm{R}}_{\text {min }}\right)$.

\section{Improving the Efficiency of DPCR-ST}

We propose three different heuristic techniques, each of which sequentially generates only $0 \leq k \leq n$ spanning trees for its input. Using smaller $k$ will reduce the time complexity of the algorithm.

For a given graph $\mathrm{G}(\mathrm{V}, \mathrm{E})$, we first compute link weight $\mathrm{w}_{i}$ for each $e_{i} \in E$ using one of three different criteria, (i) CR1: $\mathrm{w}_{i}=\mathrm{c}_{i} / \mathrm{r}_{i}$, (ii) CR2: $\mathrm{w}_{i}=\mathrm{c}_{i}$, and (iii) $\mathrm{CR} 3: \mathrm{w}_{i}=-\left(\log \mathrm{r}_{i}\right)$. Then, for each criterion, we use a modified Prim's algorithm [10] to sequentially generate all spanning trees of $G$, sorted in their increasing weights. Note that the weight of a spanning tree is calculated as the sum of the weight of each link in the spanning tree. As an example, we obtain the following orders for the spanning trees in Table I; CR1: $\left(\mathrm{ST}_{2}, \mathrm{ST}_{7}, \mathrm{ST}_{1}, \mathrm{ST}_{3}\right.$, $\left.\mathrm{ST}_{6}, \mathrm{ST}_{8}, \mathrm{ST}_{4}, \mathrm{ST}_{5}\right), \mathrm{CR} 2:\left(\mathrm{ST}_{7}, \mathrm{ST}_{2}, \mathrm{ST}_{1}, \mathrm{ST}_{8}, \mathrm{ST}_{6}, \mathrm{ST}_{4}, \mathrm{ST}_{5}\right.$, $\left.\mathrm{ST}_{3}\right)$ and CR3: $\left(\mathrm{ST}_{3}, \mathrm{ST}_{2}, \mathrm{ST}_{5}, \mathrm{ST}_{6}, \mathrm{ST}_{1}, \mathrm{ST}_{8}, \mathrm{ST}_{4}, \mathrm{ST}_{7}\right)$. Note that (3) to (6) consider spanning trees starting from $\mathrm{ST}_{n}$, and thus DPCR-ST sets $\mathrm{ST}_{n}$ as the least weighted spanning tree, $\mathrm{ST}_{n-1}$ as, the second least weighted, etc. Since Yen's algorithm requires a time complexity of $O(k \times|\mathrm{V}| \times(|\mathrm{E}|+|\mathrm{V}| \times \log |\mathrm{V}|)), \quad$ DPCR-ST requires an extra $O(n \times|\mathrm{V}| \times(|\mathrm{E}|+|\mathrm{V}| \times \log |\mathrm{V}|)) \quad$ time complexity for the improvement, i.e., $\quad O\left(\psi \times b \times|\mathrm{V}|^{4}+n \times|\mathrm{E}| \times \check{\mathrm{R}}_{\text {min }}+\right.$ $n \times|\mathrm{V}| \times(|\mathrm{E}|+|\mathrm{V}| \times \log |\mathrm{V}|))$. Note that our DPCR-ST generates only the first $k$ least weight spanning trees. Thus, this improvement does not require all spanning trees a priori, which improves DPCR-ST's time complexity, i.e., $O\left(\psi \times b \times|\mathrm{V}|^{4}+n \times|\mathrm{E}| \times \check{\mathrm{R}}_{\text {min }}+k \times|\mathrm{V}| \times(|\mathrm{E}|+|\mathrm{V}| \times \log |\mathrm{V}|)\right)$.

\section{SimUlation AND DisCUSSION}

We have implemented our DPCR-ST in C language to generate the topology of the 76 fully connected networks in [5] with the number of nodes, links and spanning trees range from 6 to 11,15 to 55 , and 1269 to $2.3 \times 10^{9}$, respectively. We obtained 76 cost matrices from the authors in [5], and use them for all link costs of all networks; the authors [5] randomly generated the integer costs with values between 1 and 100. Like in [5], we set $R_{\min }$ to either 0.9 or 0.95 and equal link reliability with value of either 0.9 or 0.95 . All simulations using DPCR-ST were run on Intel Core i5 with $2.53 \mathrm{GHz}$ with $4 \mathrm{~GB}$ of RAM, running Linux (Ubuntu Core 11.10).
For each of the 76 fully connected network topologies in [5],we first generated its spanning trees in four different orders: random, CR1, CR2, and CR3, described in Section IV.D. We have used Prim's algorithm [10] to generate the randomly ordered spanning trees, and modified the algorithm to generate the spanning trees for the three sorted criteria. Then, we used DPCR-ST on each set of spanning trees to generate its feasible topology with minimum cost. Each of the $76 C_{\text {best }}$ is the minimum among the costs of topologies generated using random, CR1, CR2, CR3, and column Rel stores its reliability.

\section{A. The Effect of Spanning Tree Orderings on the Performance of DPCR-ST.}

DPCR-ST with random ordered spanning trees generates $C_{\text {best }}$ only in 28 of 76 networks (36.8\%), which is the worst as compared to CR1 (82.8\%), CR2 (63.1\%), and CR3 (72.3\%). Further, for each case in which the random order generates $C_{\text {best }}$, at least one of the other three orders was also able to produce the result. This result shows the merit of pre-ordering spanning trees for our DP approach.

To compare the performances of CR1, CR2, and CR3, we summarize their results in Table II and III. The tables show the total number of topologies generated with cost $\mathrm{Cbes}_{t}$ and their cost optimality with respect to $C_{\min }$ - the cost of $\mathrm{G}_{m i n}$, i.e., $C_{\text {best }}>C_{\text {min }}, \quad C_{\text {best }}=C_{\text {min }}, C_{\text {best }}<C_{\text {min }}$. Note that $C_{\text {min }}$ is the minimum cost of each topology with reliability at least $R_{\min }$ as reported in [5]. As stated in [2], the reliability of each topology with cost $C_{\min }$ was estimated using a Monte Carlo method that produces result within $1 \%$ of $R_{\text {min }}$.

As shown in Table II, CR1 is the best performer, producing $C_{\text {best }} 82.8 \%$ of the time, followed by CR3 with $72.3 \%$ and CR2 with 63.1\%; see column "Total". For each order, the last column in the table shows the total number of topologies with $\operatorname{cost} C_{\text {best }}$ that can only be generated using its two alternative sorting criteria; e.g., row 1 of the table shows that CR1 produces 13 topologies with cost worse than that produced using CR2 and/or CR3.

TABLE II: COMPARISONS AMONG CR1, CR2 AND CR3

\begin{tabular}{|c|c|c|c|c|c|}
\hline \multirow{2}{*}{ Cost } & \multicolumn{4}{|c|}{ Total number of topologies with cost $C_{\text {best }}$} & \multirow{2}{*}{$\begin{array}{l}\text { Total number of } \\
\text { topologies with } \\
\text { cost } C_{\text {best }} \text { using } \\
\text { the other two } \\
\text { sorting criteria }\end{array}$} \\
\hline & $\begin{array}{l}C_{\text {best }}< \\
C_{\text {min }}\end{array}$ & $\begin{array}{c}C_{\text {best }}= \\
C_{\min }\end{array}$ & $\begin{array}{l}C_{\text {best }}> \\
C_{\text {min }}\end{array}$ & Total & \\
\hline CR1 & $\begin{array}{c}27 \\
35.5 \% \\
\end{array}$ & $\begin{array}{c}26 \\
34.2 \% \\
\end{array}$ & $\begin{array}{c}10 \\
13.1 \% \\
\end{array}$ & $\begin{array}{c}63 \\
82.8 \% \\
\end{array}$ & $\begin{array}{c}13 \\
17.2 \% \\
\end{array}$ \\
\hline CR2 & $\begin{array}{c}17 \\
22.3 \% \\
\end{array}$ & $\begin{array}{c}24 \\
31.5 \% \\
\end{array}$ & $\begin{array}{c}7 \\
9.2 \% \\
\end{array}$ & $\begin{array}{c}4863.1 \\
\%\end{array}$ & $\begin{array}{c}28 \\
36.8 \%\end{array}$ \\
\hline CR3 & $\begin{array}{c}25 \\
32.8 \%\end{array}$ & $\begin{array}{c}24 \\
31.5 \%\end{array}$ & $\begin{array}{c}6 \\
7.8 \%\end{array}$ & $\begin{array}{c}55 \\
72.3 \%\end{array}$ & $\begin{array}{c}21 \\
27.6 \%\end{array}$ \\
\hline
\end{tabular}

As shown in Table II, our DPCR-ST can produce topology with $C_{\text {best }}<C_{\text {min }}$ because we round off each reliability to its closest integer and use a Monte Carlo method [9] that computes reliability within $0.5 \%$ of $R_{\text {min }}$. The table also shows that CR1, CR2, and CR3 produce $69.7 \%, 53.8 \%, 64.3 \%$ of topologies with cost less or equal than $C_{\min }$, respectively. Thus, in term of optimality, CR1 (CR2) is the best (worst) performer.

Table III shows the total number of $C_{\text {best }}$ uniquely produced using one or more of the three different ordering criteria. The table shows that there are in total 8,12 and 1 topology with cost $\mathrm{C}_{\text {best }}$ uniquely generated by CR1, CR2 and 
CR3, respectively, and the three criteria produce the same topologies $35 / 76=46 \%$ of the time. Further, there are 1 and 19 topologies that can only be generated by either CR1 or CR2 and CR1 or CR3, respectively. The results show that it is important for DPCR-ST to use the three ordering criteria, CR1, CR2 and CR3, and select the best among their results to generate topologies with lower cost. As shown in the table, such approach produces only $18.4 \%$ topologies with less optimal cost.

TABLE III: Distribution OF CBest Generated Using OnE OR MoRE CRITERIA

\begin{tabular}{|c|c|c|c|}
\hline Cost & $C_{\text {best }}<C_{\text {min }}$ & $C_{\text {best }}=C_{\text {min }}$ & $C_{\text {best }}>C_{\text {min }}$ \\
\hline Order & & & 4 \\
\hline CR1 & 3 & 1 & 4 \\
\hline CR2 & 2 & 6 & 0 \\
\hline CR3 & 1 & 0 & 0 \\
\hline CR1,CR2 & 0 & 1 & 3 \\
\hline CR1,CR3 & 9 & 7 & 0 \\
\hline CR2,CR3 & 0 & 0 & 3 \\
\hline CR1,CR2,CR3 & 15 & 17 & $14(18.4 \%)$ \\
\hline Total & $30(39.4 \%)$ & $32(42.1 \%)$ & \\
\hline
\end{tabular}

\section{B. DPCR-ST versus Existing Approaches}

Table IV compares the effectiveness of our DPCR-ST against four state-of-the-art approaches, i.e., NGA [2], LS/NG [3], ACO-SA [5], and BDD [11], using the 76 fully connected networks. Since we were unable to obtain the source codes for the four approaches in [2], [3], [5], and [11], we have used their reported results in our comparisons.

As shown in Table IV, while NGA, LS/NGA and ACO-SA could generate optimal solutions for 16, 24 and 48 out of 76 instances, respectively, and BDD obtain optimal solutions for 14 out of 45 instances, DPCR-ST could produce 62 out of 76 optimal results $(81.5 \%)$, significantly improving the effectiveness of the existing algorithms.

TABLE IV: COMPARISON BETWEEN DPCR-ST, NGA, LS/NGA, ACO_SA AND BDD

\begin{tabular}{|c|c|c|c|c|c|}
\hline & DPCR-ST & NGA & LS_GA & ACO_SA & BDD \\
\hline$C_{\text {best }}=C_{\min }$ & $\begin{array}{c}32 \\
(42.1 \%)\end{array}$ & $\begin{array}{c}16 \\
(21 \%)\end{array}$ & $\begin{array}{c}24 \\
(31.5 \%)\end{array}$ & $\begin{array}{c}48 \\
(63.1 \%)\end{array}$ & $\begin{array}{c}14 \\
(33 \%)\end{array}$ \\
\hline$C_{\text {best }}<C_{\min }$ & $\begin{array}{c}30 \\
(39.4 \%)\end{array}$ & N/A & N/A & N/A & NA \\
\hline
\end{tabular}

Further, 30 of $62 C_{\text {best }}$ generated using DPCR-ST are better than $C_{\text {min }}$. These results show the superiority of our efficient DP approach as compared to the existing state-of-the-arts solutions [2], [3], [5], and [11].

\section{CONCLUSION}

We have defined a network topology design problem, NTD-CR, to generate topology that has the minimum cost subject to a reliability constraint $R_{\text {min }}$. We have proposed a heuristic based on dynamic programming, to solve NTD-CR. Our method DPCR-ST incrementally generates only a selected $k$ spanning trees from the network, and is scalable on networks having large number of spanning trees. We have proposed to sort the spanning trees using three different orders to optimize our method's effectiveness and efficiency. The experimental study shows that the DPCR-ST approach is able to generate $81.5 \%$ optimal solutions. We plan to design an alternative DP approach that heuristically deletes links from the original topology to find an optimal design.

\section{REFERENCES}

[1]. A. Kumar, R. Pathak, and Y. Gupta, "A genetic algorithm for distributed system topology design," Computers and Industrial Engineering, vol. 28, pp. 659-670, 1995.

[2]. B. Dengiz, F. Altiparmak, and A. Smith, "Efficient optimization of all-terminal reliable networks," IEEE Trans. on Reliability, vol. 41, no. 1, pp. 18-26, 1997.

[3]. B. Dengiz, F. Altiparmak, and A. Smith, "Local search genetic algorithm for optimal design of reliable networks," IEEE Trans. on Evolutionary Computation, vol. 1, no. 3, pp. 179-188, 1997.

[4]. C. Papagianni and K. Papadopoulos, "Communication Network Design Using Particle Swarm Optimization," in Proc. International Multi Conference on Computer Science and Information Technology, Poland, 2008, pp. 915-920.

[5]. F. Altiparmak, B. Dengiz, O. Belgin, "A hybrid Ant Colony Optimization approach for the Design of Reliable Networks," IIE Transactions, vol. 42, pp. 273-287, 2010.

[6]. M. Desjarlais and R. Molina, "Counting spanning trees in grid graphs,"Congressus Numerantium, vol. 145, pp. 177-185, 2000.

[7]. S. Martello, D. Pisinger, P. Toth, "Dynamic programming and strong bounds for the 0-1 knapsack problem," Management Science, vol. 45, pp. 414-424, 1999.

[8]. S. Rai, and S. Soh, "CAREL: Computer aided reliability evaluator for distributed computer networks," IEEE Trans. Parallel and Distributed Systems, vol. 2, pp. 199-213, 1991.

[9]. S. Yeh, J. Lin, W. Yeh, "New Monte Carlo method for estimating network reliability," in Proc. the 16th International Conference on Computers and Industrial Engineering, Japan, 1994,pp. 723-726.

[10]. Snippets Dzone. [Online]. Available: http://www.dzone.com/snippets/prims-algorithm-finding.

[11]. G. Hardy, C. Lucet, and N. Limnios, "A BDD-based heuristic algorithm for design of reliable networks with minimal cost," in Proc. International Conference on Mobile Ad Hoc and Sensor Networks, China, 2006, pp.13-15.

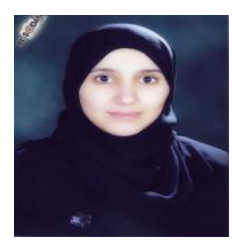

Basima Elshqeirat received the BS (2004), and MS (2008) degrees in computer science from the University of Jordan, Jordan. She is currently working toward the Ph.D degree in Computer Science at Curtin University. Her research interests include network reliability and network design.

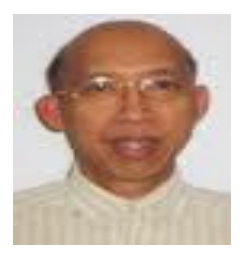

Sieteng Soh received a B.S. degree in electrical engineering from the University of Wisconsin, Madison, and M.S. and Ph.D in electrical engineering from the Louisiana State University, Baton Rouge. He was a faculty member (1993-2000), and the director of the Research Institute (1998-2000) at Tarumanagara University-Indonesia. He is currently a Senior Lecturer with the Department of Computing at Curtin University. Dr. Soh has published papers in refereed international journals, and conference proceedings in the area of computer network, network reliability, and parallel and distributed processing. He is a member of the IEEE.

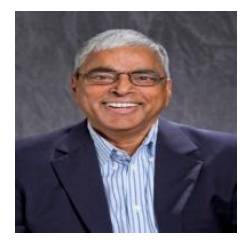

Suresh Rai received the $\mathrm{PhD}$ degree in electronics and communication engineering from Kurukshetra University, Kurukshetra, Haryana, India, in 1980. $\mathrm{He}$ is currently a professor with the Division of Electrical and Computer Engineering, School of Electrical Engineering and Computer Science at Louisiana State University, Baton Rouge. His research interests include network traffic, wavelet-based compression, watermarking in audio and video, and network reliability and security. He is a senior member of the IEEE and a member of the IEEE Computer Society.

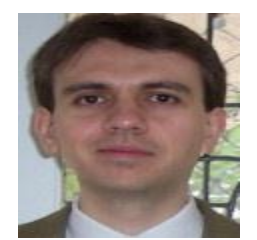

Mihai Lazarescu received his B.S. (Computer Science) degree with First Class Honoursin 1996, and his Ph.D in computer Science from the Curtin University, in 2000. He has been a senior member of the IMPCA research institute for 10 years and is currently the head of Department of Computing and an associate professor at Curtin University. He has published over 60 papers in refereed international journals, and conference proceedings in the areas of artificial intelligence, machine vision, data mining and network reliability. 